\title{
Psicoterapia como estratégia de tratamento dos transtornos alimentares: análise crítica do conhecimento produzido
}

\author{
Use of psychotherapy as strategy for treatment \\ of eating disorders: critical analysis \\ of scientific knowledge
}

\author{
Fabio SCORSOLINI-COMIN \\ Manoel Antônio dos SANTOS
}

\begin{abstract}
Resumo
O objetivo deste estudo foi empreender uma análise crítica acerca do conhecimento científico produzido sobre a utilização das psicoterapias como estratégia de tratamento dos transtornos alimentares. A partir de buscas nas bases PsycINFO, PePSIC e SciELO, no período entre 1999 e 2011, foram recuperados 35 artigos, categorizados em: psicoterapias breves, grupais, psicodinâmicas, complementares, bem como psicoterapias aliadas a outros tratamentos, como o psicofarmacológico. As abordagens mais frequentemente mencionadas foram psicodinâmicas e cognitivo-comportamentais. A modalidade de atendimento predominante foi a grupal. Ainda que preconizando o uso combinado de diversas estratégias, a literatura é unânime em destacar a importância das psicoterapias no tratamento. A análise crítica evidenciou necessidade de leituras que transcendam a mera identificação de técnicas psicoterápicas consideradas mais eficazes para o tratamento dos transtornos alimentares. Deve-se considerar o contexto mais amplo no qual os tratamentos são propostos, bem como promover um diálogo aberto entre enfoques teóricos, valorizando a pluralidade de saberes e a psicoterapia como prática em permanente transformação.
\end{abstract}

Unitermos: Anorexia nervosa. Bulimia. Psicoterapia. Transtornos alimentares.

\begin{abstract}
The objective of this study was to conduct a critical analysis of scientific knowledge on the use of psychotherapy as strategy for treatment of eating disorders. From searches in the databases PsycINFO, PePSIC and SciELO in the period 1999-2011, 35 articles were retrieved in full, categorized as brief psychotherapy, group, psychodynamic, alternative psychotherapy, and psychotherapy combined with other treatments such as those of a pharmacological nature. The most mentioned approaches were the psychodynamic and cognitive-behavioral types. The predominant type of service was the group. Although the use of a combination of different strategies is recommended, the literature is unanimous in pointing out the importance of psychotherapy in treatment. The critical analysis showed the need for reading that transcends the mere identification of psychotherapeutic techniques considered most effectivefor the treatment of eating disorders. The broader context in which treatments are offered must be considered, as well as promoting an open dialogue between theoretical approaches, prioritizing the plurality of knowledge and the relevance of psychotherapy as a practice that changes constantly.
\end{abstract}

Uniterms: Anorexia nervosa. Bulimia. Psychotherapy. Eating disorders.

\section{$\boldsymbol{\nabla \nabla \nabla \nabla}$}

1 Universidade Federal do Triângulo Mineiro, Instituto de Educação, Letras, Artes, Ciências Humanas e Sociais, Departamento de Psicologia. Av. Getúlio Guaritá, 159, Abadia, 38025-440, Uberaba, MG, Brasil. Correspondência para/Correspondence to: F. SCORSOLINI-COMIN. E-mail: <scorsolini_usp@yahoo.com.br>.

2 Universidade de São Paulo, Faculdade de Filosofia, Ciências e Letras de Ribeirão Preto, Departamento de Psicologia. Ribeirão Preto, SP, Brasil. 
É cada vez mais crescente a visibilidade social conferida aos Transtornos Alimentares (TA), dentre os quais se destacam, pela prevalência e relevância clínica, a Anorexia Nervosa (AN) e a Bulimia Nervosa (BN). Além disso, os TA estão cada vez mais ocupando espaço na mídia. Os significativos índices de mortalidade que acompanham os TA têm colocado esses quadros como importantes problemas de saúde pública (American Psychiatric Association - APA, 2011; Andrade \& Santos, 2009; Scorsolini-Comin, Souza \& Santos, 2010; Weinberg \&Berlinck, 2010).

Segundo os critérios do Diagnostic and Statistical Manual of Mental Disorders - IV Revised (DSM-IV-R) (American Psychiatric Association, 2000), a AN é caracterizada, entre outros requisitos, pela recusa na manutenção do peso corporal saudável, medo intenso do ganho de peso - ou de se tornar obeso -, e distorção da imagem corporal. Já a BN é caracterizada por episódios recorrentes de compulsão alimentar, comportamentos purgativos com frequência de ao menos três vezes por semana, e autoavaliação excessivamente influenciada pelo próprio peso e forma física (Souza, 2011).

Na literatura especializada os TA são muitas vezes caracterizados como quadros que se alinham às denominadas "patologias do vazio", que na verdade seriam transtornos mentais que definiriam os modos de subjetivação contemporâneos (Peres \& Santos, 2011). É comum que os pacientes com TA associem a instalação dos sintomas com uma reação a um evento estressor, como o término de relacionamento amoroso ou a perda de ente querido (Sicchieri, Santos, Dos Santos \& Ribeiro, 2007; Souza \& Santos, 2010). Desse modo, o meio social (família, escola, redes de contato) estaria diretamente relacionado não apenas ao aparecimento dos sintomas iniciais dos TA, mas também a sua evolução e perpetuação. Muitos são os tratamentos empregados nesse contexto, como o uso de psicofármacos, psicoterapia individual e grupal, terapia familiar, grupo multifamiliar, aconselhamento nutricional e internação quando necessário (APA, 2011; Araújo \& Henriques, 2011; Gowers \& Bryant-Waugh, 2004; Grothaus, 1998; Souza, 2011). Dentre as estratégias de tratamento preconizadas, a prática psicoterápica tem sido referida como importante recurso no acompanhamento dos pacientes (Sicchieri et al., 2007; Weinberg \& Berlinck, 2010).

As psicoterapias são consideradas práticas de 852 atenção psicológica que visam a auxiliar o indivíduo a lidar com seu sofrimento emocional. Na vertente psicanalítica, o sofrimento pode ser resultante da dificuldade em recrutar mecanismos de defesa saudáveis diante dos conflitos psíquicos, o que pode gerar padrões adaptativos problemáticos que comprometam o funcionamento da personalidade (Santos, 2007). As psicoterapias têm sido reconhecidas como uma das formas prioritárias para a resolução de problemas de saúde mental, ao lado de técnicas e procedimentos empregados pela psicofarmacoterapia, reabilitação psicossocial e profissional, dentre outras (Organização Mundial da Saúde - OMS, 2002; Santeiro, 2008). Embora essa reflexão da OMS acerca do emprego da psicoterapia pareça nova, deve-se destacar que as psicoterapias são procedimentos sistemáticos e cientificamente fundamentados, cuja inscrição histórica é relativamente recente, já que remonta a pouco mais de um século. Trata-se de campo fragmentado e multifacetado, atravessado por uma pluralidade de sistemas teóricos e abordagens, embora apenas cerca de duas dezenas delas sejam dominantes (Hanns, 2004; Santos, 2007; Weissmann, Markowitz \& Klerman, 2009).

Investigando as bases de dados bibliográficas nacionais, Pinto, T.V. Santeiro e F.R.M. Santeiro (2010) destacaram que os enfoques teóricos mais utilizados são os de base psicanalítica, seguidos pelo enfoque cognitivo-comportamental e pela grupoanálise. Dentre as demais abordagens existentes, porém com menor representatividade, destacaram-se as de matriz fenomenológico-existencial, gestalterapia, psicoterapia analítica, terapia sistêmica, construcionismo social, psicodrama, logoterapia e o referencial sócio-histórico. Em termos das psicopatologias investigadas a partir do emprego de técnicas psicoterápicas, os TA e a obesidade ocupam a segunda colocação, vindo atrás apenas dos transtornos de ansiedade (transtorno de pânico, transtorno de ansiedade social e transtorno de stress pós-traumático).

Investigar a produção científica nacional e internacional acerca das psicoterapias constitui um desafio (Barth, 1991; Leichsenring, 2005; Messer, 2001; Pinto et al., 2010; Santos \& Zaslavsky, 2007), devido à diversidade de categorias de análise necessárias a uma compreensão mais global do fenômeno. O campo é vasto, e a análise empreendida deve necessariamente contemplar sua complexidade em termos de proposta teórica, 
enquadre, duração, manejo de processo terapêutico, perfil da clientela atendida, formação e capacitação profissional do psicoterapeuta, dentre outras variáveis. Também é preciso analisar criticamente a qualidade científica dos estudos divulgados. No contexto do tratamento dos TA, o mapeamento da literatura científica pode tanto instrumentalizar a prática do psicoterapeuta como apontar a pluralidade das contribuições oferecidas pelos diferentes estudos. Pode, ainda, apontar as lacunas existentes no conhecimento produzido, sugerindo novas questões de pesquisa para gerar futuras investigações. Desse modo, na realização de um estudo de revisão sistemática de literatura, há que se primar por um percurso metodológico que abarque essas dificuldades e que possa convertê-las em desafios passíveis de investigação.

O objetivo deste estudo foi empreender uma análise crítica acerca do conhecimento científico produzido sobre a utilização das psicoterapias como estratégia de tratamento dos TA, notadamente da AN e BN.

\section{Método}

Trata-se de uma revisão sistemática de literatura. Estudos dessa natureza são relevantes por contribuírem para delimitar a produção existente em um determinado contexto (nacional e/ou internacional), permitindo que se apontem lacunas, limites e também possibilidades de novas investigações, que contemplem a temática de interesse de modo mais completo e aprofundado. Desse modo, não basta que se aponte a produção existente e suas características, mas também que se lance ao acervo constituído uma visão crítica acerca do processo de construção do conhecimento na área (Biasoli-Alves, 1998; Creswell, 2010; Scorsolini-Comin, 2010; Toffoli \& Ferreira, 2011).

Além disso, os artigos revisados permitem compreender que a pertinência e efetividade do trabalho intelectual do pesquisador dependem da forma como é feita a comunicação com seus leitores, informando e disseminando os resultados de suas pesquisas, bem como animando nos meios científicos discussões que possam não apenas fazer o conhecimento avançar na área, como subsidiar diretamente a prática assistencial.

Como o objetivo da revisão foi recuperar publicações desenvolvidas no contexto nacional e interna- cional, prioritariamente ligadas à Psicologia, foram consultadas as bases PsycINFO, PePSIC e SciELO.

Nesta revisão foram selecionados apenas artigos indexados, visto que esse tipo de produção científica passa por um rigoroso processo de avaliação por pares, conforme sugerido pelas orientações de Scorsolini-Comin (2010). Sendo assim, foram excluídos artigos não indexados, livros, capítulos de livros, teses, dissertações, monografias, resenhas, cartas e notícias. Foram incluídos artigos concernentes ao assunto abordado, sem restrição de idioma. Foram descartados aqueles que não se relacionavam ou não se aproximavam do tema psicoterapia e transtorno alimentar, ou que trouxessem apenas uma abordagem periférica da intervenção terapêutica.

O período selecionado foi de 1999 a 2011, buscando-se priorizar a produção nacional e internacional recente acerca do tema. A revisão não se restringiu apenas à seleção de artigos produzidos no campo da Psicologia, haja vista que as psicoterapias frequentemente estabelecem interfaces com outros campos do saber, como Medicina, Ciências Sociais, Enfermagem, Nutrição eTerapia Ocupacional, entre outros. A partir dessa consideração, optou-se por não se delimitar previamente a área na qual os estudos foram publicados, priorizando, assim, o diálogo inter e multidisciplinar.

\section{Procedimentos}

A etapa de levantamento de artigos ocorreu entre agosto e setembro de 2011. Foram utilizados os descritores: psicoterapia, transtornos alimentares, anorexia nervosa, bulimia, bem como as seguintes combinações: psicoterapia "and" transtornos alimentares, psicoterapia "and" bulimia, psicoterapia "and" anorexia nervosa, todos em português e em inglês. Definiu-se que os descritores deveriam aparecer no título e/ou no resumo dos artigos. Há que se considerar que os quatro unitermos utilizados (psicoterapia, transtornos alimentares, bulimia e anorexia nervosa) fazem parte da terminologia tanto da Psicologia como das Ciências da Saúde, conforme busca no portal da Base Virtual em Saúde (Terminologia Psi Alfabética e DeCS - Descritores em Ciências da Saúde).

Primeiramente, foi realizada uma leitura minuciosa dos resumos encontrados a partir desses uniter- 
mos, excluindo-se os artigos repetidos e os que não se enquadravam nos critérios de inclusão elencados. Nessa seleção preliminar, realizada a partir de minucioso exame dos resumos, os artigos selecionados foram recuperados e lidos na íntegra. Posteriormente, em função da maior ou da menor proximidade com o tema, uma nova seleção foi realizada, restringindo-se a revisão apenas aos estudos diretamente relacionados à psicoterapia e aos TA. Desse modo, foram considerados para discussão apenas os artigos recuperados nessa última etapa da seleção, os quais constituíram o corpus de análise.

O material foi organizado em categorias temáticas, segundo as modalidades de psicoterapia (individual, grupal ou familiar, por exemplo) e abordagens (psicodinâmica ou cognitivo-comportamental, por exemplo), de modo a facilitar a visualização das características mais salientes, bem como dos limites e potencialidades do material recuperado. Há que se destacar, no entanto, que tal organização não excluiu a possibilidade de que um mesmo artigo fosse cotejado em mais de uma categoria, haja vista a necessidade de não apenas caracterizar e classificar a produção recente, mas também propor uma discussão crítica de sua qualidade científica. Para assegurar uma discussão ampliada dos resultados de pesquisas, nem todos os estudos foram sistematicamente apresentados em suas características individuais, mas contemplados nas reflexões desenvolvidas por categorias e na discussão final.

\section{Resultados e Discussão}

Foram encontradas 1664 publicações, de acordo com a temática e os descritores elencados. Posteriormente, aplicando-se os critérios de inclusão/exclusão adotados, 1629 estudos foram descartados por não serem pertinentes ao assunto investigado (principalmente, por tratar dos TA sem qualquer vinculação com a psicoterapia ou abordar a psicoterapia e elencar o TA apenas como um exemplo, dentre outros, de problemática de saúde mental na qual se aplica o dispositivo psicoterapêutico) ou, ainda, por se referirem a livros, capítulos, teses, dissertações, monografias, resenhas ou notícias. Sendo assim, após minucioso trabalho de depuração, a amostra final foi composta por 35 artigos, que foram lidos na íntegra e analisados em detalhe, 854 segundo os critérios propostos. Após análise criteriosa, os conteúdos dos artigos foram categorizados em eixos de análise, que serão apresentados a seguir, de modo a facilitar a compreensão dos dados.

\section{Psicoterapias breves $(n=3)$}

Essa categoria abarca os artigos que priorizaram as psicoterapias breves no tratamento dos TA, independentemente da abordagem teórica utilizada. Fassino, Amianto e Ferrero (2008) descrevem que a psicoterapia breve está ganhando cada vez mais interesse no mundo inteiro, devido à relação favorável entre custo e eficácia. Os autores empregaram a psicoterapia psicodinâmica breve adleriana, que parte do pressuposto de que o indivíduo representa uma unidade psicossomática integrada no contexto social. Os objetivos dessa abordagem são: solucionar o problema (ou parte dele), reduzir os sintomas e promover um aumento global da qualidade de vida do paciente. O esquema de tratamento, que inclui 15 sessões de psicoterapia breve, foi aplicado como abordagem psicoterapêutica em alguns estudos sobre TA e transtornos de personalidade grave, exibindo boa eficácia. A psicoterapia psicodinâmica breve é também abordada no estudo de Lewis, Dennerstein e Gibbs (2008), embora não sejam conclusivos os estudos que empregam essa modalidade de tratamento no contexto dos TA.

Fassino, Daga, Pierò e Rovera (2002) investigaram o fenômeno do abandono de psicoterapia breve na AN. Pacientes que abandonaram o tratamento mostraram níveis mais elevados de raiva e pontuações mais baixas para as dimensões de caráter (baixa auto-orientação e baixo cooperativismo). Não foram encontradas diferenças estatisticamente significantes entre os dois grupos em relação às variáveis sociodemográficas e clínicas. Concluiu-se que o abandono de psicoterapia breve parece estar relacionado a aspectos psicopatológicos de personalidade (como a tendência para reprimir a raiva, estratégia defensiva que é encontrada também nos distúrbios psicossomáticos) e à presença de dimensões mais comprometidas da personalidade (como nos casos de comorbidades com transtornos de personalidade).

De acordo com os estudos encontrados, as psicoterapias breves podem ser compreendidas como um tratamento adequado para os TA, embora não sejam 
relatadas com maior frequência na literatura científica. A escassez de estudos disponíveis sugere a necessidade de que outras intervenções que se utilizam desse enfoque sejam trazidas para discussão, a fim de ampliar o repertório de experiências psicoterapêuticas bem-sucedidas no contexto dos TA, ou mesmo para refutar a efetividade de seu emprego, delimitando assim os limites de sua aplicabilidade.

\section{Psicoterapias grupais $(n=5)$}

Nessa categoria é conferida visibilidade às práticas psicoterapêuticas grupais no tratamento, já referidas em estudos anteriores como bastante difundidas no contexto dos TA (Fernandes, 2006; Souza, 2011). A psicoterapia de grupo foi alvo de diversos estudos encontrados nesta revisão, como os de Moro (2001) e Salierno (2001). A investigação de Sydow, Beher, Schweitzer e Retzlaff (2010) utilizou a terapia sistêmica como abordagem psicoterápica. Uma metanálise de conteúdo foi realizada para analisar a eficácia da terapia sistêmica para o tratamento de transtornos mentais na idade adulta. Foram incluídos 38 ensaios Clínicos Controlados e Randomizados (RCT) na avaliação dos sistemas de terapia orientados em várias configurações (família, casal, individual, de grupo, terapia de grupo multifamiliar). Dados derivados de 34 estudos permitiram concluir que essa abordagem mostrou-se eficaz para o tratamento dos TA.

Segundo dados da revisão realizada por Lock (2010), embora os TA sejam transtornos psiquiátricos comuns no início da adolescência, são poucos os tratamentos baseados em evidências disponíveis nessa faixa etária. Revisão crítica de ensaios clínicos randomizados constatou apenas sete estudos publicados com psicoterapia para AN em adolescentes, em um total de 480 investigações revisadas. Foram identificados apenas dois estudos publicados sobre psicoterapia ambulatorial para adolescentes com BN, em um total de 165 artigos. Três estudos recuperados na referida revisão sugerem que a terapia familiar oferece mais resultados de sucesso do que a de base individual. A terapia familiar parece ser, segundo a literatura, superior à psicoterapia individual nesses quadros. Apontamentos semelhantes foram obtidos no estudo de Ruiz-Lázaro (2003).

Desse modo, as intervenções grupais podem ser consideradas uma modalidade não apenas de uso frequente no plano de tratamento dos TA, como também a forma predominante de atendimento nos sistemas públicos de saúde. Considerando essa última constatação, há que se destacar a necessidade de que os grupos sejam realizados não apenas como uma forma de lidar com a demanda crescente nesses serviços, mas com propósitos específicos que coloquem o atendimento grupal como essencial no apoio aos pacientes e familiares, conforme destacado nos estudos de Souza e Santos (2010) e de Tasca, Ritchie e Balfour (2011).

\section{Abordagens psicodinâmicas $(n=8)$}

Nessa categoria foram priorizadas as abordagens psicodinâmicas, fundamentadas em diferentes teóricos da Psicanálise e sistematizadas em diferentes modalidades de atendimento. Zerbe (2001) considera que as intervenções psicodinâmicas podem fornecer um caminho ímpar para o tratamento, pois desempenham um papel crucial para a compreensão e tratamento de pacientes com TA. Os antecedentes de desenvolvimento do transtorno são esmiuçados, fornecendo um ambiente seguro para que o paciente possa elaborar sua história pessoal. No referido estudo, as intervenções e estratégias se resumem a auxiliar os médicos a intervirem de forma mais eficaz em vários dos problemas mais desconcertantes que confrontam os terapeutas no contexto dos TA. Esses desafios incluem o processamento da identificação projetiva, por meio da avaliação das reações transferenciais e contratransferenciais mais comuns, incentivando o paciente a gerir de modo eficaz um leque de conteúdos emocionais não expressos verbalmente.

Teriato (2009) destaca que uma característica peculiar à psicoterapia de adolescentes que desenvolveram TA é que a intervenção é mais eficaz durante a fase inicial do tratamento, quando os sintomas podem fornecer pistas sobre a jornada de desenvolvimento do jovem paciente. Selecionando aquelas contribuições clínicas que se relacionam com o aparecimento dos TA como uma psicopatologia da identidade, o autor sugere que é importante concentrar-se nas fases do processo terapêutico que são cruciais na construção de um espaço de intimidade e aproximação com os conteúdos internos, o que pode ser utilizado pelo adolescente para crescer e descobrir-se a si mesmo. Em particular, definir 
claramente o contrato terapêutico é considerado fundamental na aplicação dessa estratégia, tendo em vista a necessidade de consolidar uma possível aliança terapêutica. A forma como o contrato é definido tanto pode contribuir para abrir como para fechar o caminho que poderia ser trilhado no percurso terapêutico. De acordo com uma orientação generalizada da literatura psicanalítica, principalmente amparada no referencial winnicottiano, o referido autor sublinha a importância de focalizar a atenção terapêutica inicial no medo dos adolescentes com TA de perderem o controle sobre o peso, que na verdade traduz o temor de descontrole dos próprios impulsos.

Tasca, Ritchie e Balfour (2011) relacionaram os resultados de pesquisas sobre apego eTA para sugerirem uma estrutura para avaliação e tratamento do funcionamento da relação de apego em pacientes que apresentam esses transtornos. Os resultados indicaram que indivíduos com padrão de apego inseguro tendem a se beneficiar menos das terapias focadas no sintoma. Segundo os autores, avaliar esses domínios do funcionamento de apego pode orientar intervenções mais focalizadas e com maior probabilidade de sucesso. São apresentados exemplos clínicos para ilustrar essa avaliação, como a psicoterapia de grupo aplicada aos TA. Dados semelhantes foram discutidos por Ramacciotti, Sorbello, Pazzagli, Vismara e Mancone (2001).

Rosa e Santos (2011) relataram a experiência de um processo terapêutico de orientação psicanalítica de longa duração com uma paciente diagnosticada com BN em comorbidade com transtorno de personalidade do tipo borderline. Em seu relato de experiência como terapeuta de pessoas com TA, Barth (2008) destacou que tais transtornos estão relacionados a problemáticas como a separação psíquica em relação à mãe e a regulação dos afetos, sendo que, muitas vezes, as pessoas com TA acabam ocultando seus sintomas na psicoterapia. Dificuldades, nessas áreas, impedem que os pacientes sejam autorreflexivos ou que utilizem o insight de maneira produtiva. Integrando essas dinâmicas, a partir de um enfoque psicanalítico, a psicoterapia focada no estabelecimento dos vínculos e do apego tende a ser bem-sucedida, fazendo com que os pacientes incrementem a confiança no terapeuta e não mais escondam seus sintomas por vergonha ou medo de rejeição. Outros estudos, como os de Fassino, Amian- to e Ferrero (2008), Bruno, Rosani e Berlincioni (2009) e Lewis, Dennerstein e Gibbs (2008), fizeram uso do enfoque psicanalítico na condução de suas intervenções, destacando resultados positivos com pacientes em diferentes estágios do tratamento, o que corrobora os apontamentos de Fernandes (2006) e é consistente com a apreciação dos TA como expressões fenomenológicas das chamadas "psicopatologias do vazio"que proliferam na era contemporânea (Peres \& Santos, 2011). Nesse sentido, as psicoterapias psicodinâmicas permitiriam ao sujeito entrar em contato com esse vazio interno, o que permitiria aberturas para a elaboração das experiências infantis catastróficas relacionadas à emergência dos sintomas de TA.

\section{Abordagens cognitivo-comportamentais $(n=8)$}

Nessa categoria são destacados os estudos que relatam práticas psicoterápicas com enfoque cognitivo-comportamental, consideradas por alguns autores como uma das abordagens mais utilizadas atualmente no tratamento dos TA no Brasil (Souza, 2011). Também no contexto internacional, Constantino, Arnow, Blasey e Stewart Agras (2005) discutem a importância do estabelecimento da aliança terapêutica no tratamento psicoterápico. A aliança é considerada pelos autores em termos da colaboração e do engajamento existentes entre paciente e terapeuta. O objetivo do estudo foi examinar a relação entre características específicas dos pacientes e o desenvolvimento da aliança terapêutica, em dois tipos diferentes de tratamento da BN: intervenção de base cognitivo-comportamental e terapia interpessoal. Os resultados obtidos com o primeiro tratamento foram mais exitosos em termos da aliança terapêutica estabelecida.

Rutherford e Couturier (2007) afirmam que as intervenções psicoterapêuticas para crianças e adolescentes com TA têm recebido, recentemente, crescente atenção por parte da literatura. Segundo revisão realizada por esses autores, houve alguns resultados muito promissores no que diz respeito a tipos específicos de tratamento para a AN, BN e Transtorno de Compulsão Alimentar Periódica (TCAP). A melhor evidência disponível sugeriu que os modelos de terapia familiar são mais eficazes para o tratamento de adolescentes com AN, e que os modelos de Terapia Cognitivo Comportamental 
(TCC) são mais eficazes para adolescentes com BN, embora a terapia familiar também possa ser utilizada com estes. Grave, Ricca e Todesco (2001) também destacaram que a TCC deve ser o tratamento inicial preferencial para o tratamento da $\mathrm{BN}$, uma vez que tal abordagem apresenta eficácia superior a outras modalidades, como a interpessoal e a psicofarmacológica.

No corpo de literatura analisado a respeito da abordagem cognitivo-comportamental, os dados comprovam a eficácia desse modelo no tratamento dos TA (Bacaltchuk \& Hay, 1999; Bowers \& Andersen, 2007; Kong, 2005; Pomerantz, 2005), em estratégia de psicoterapia associada a tratamento medicamentoso ou a outras abordagens, como a psicoterapia interpessoal (Hendricks \&Thompson, 2005).

\section{Abordagens complementares em psicoterapias $(n=10)$}

Nessa categoria foram incluídos estudos conduzidos com abordagens contemporâneas, aqui denominadas de complementares. São estratégias consideradas "alternativas" em relação aos modelos mais consagrados, por serem mencionadas em menor número na produção nacional e internacional sobre os TA, ou por não delimitarem de modo enfático a abordagem empregada - ou, então, por combinarem mais de uma abordagem. Não se está tomando aqui o termo "alternativo" em seu caráter pejorativo e depreciativo, isto é, para designar estratégias que incluam procedimentos não comprovados ou não respaldados pelo conhecimento científico.

Um exemplo é o estudo de Daini (2009), que fez uso de métodos projetivos, que são menos utilizados em comparação com os métodos de autoavaliação no estudo dos TA. O estudo destacou dois problemas principais: a presença e a qualidade da dinâmica depressiva, tanto na AN e BN como na obesidade, e a questão da identidade de gênero. Dificuldades no plano da identificação de gênero são sublinhadas, especialmente nos TA do sexo masculino. Alguns estudos longitudinais sobre mudanças terapêuticas obtidas são também discutidos pela autora, que destaca a importância da combinação de psicoterapia psicodinâmica com avaliação com base em técnicas projetivas como importantes ferramentas auxiliares no tratamento dos TA, o que deve ser aprofundado em estudos futuros. Gaudriault e Guilbaud (2005) avaliaram o desenvolvimento de mulheres com BN durante o tratamento e, em especial, durante a psicoterapia. O método de avaliação levou em conta os efeitos adversos associados com os TA em relação à autoimagem e às relações de objeto, a partir da comparação dos resultados obtidos na prova de Rorschach.

Tantillo (2004) refere a abordagem da Terapia Relacional (TR) como uma das possibilidades terapêuticas promissoras no tratamento dos TA. Um dos aspectos críticos da TR, que a distingue de outros tratamentos para TA, é o uso que o terapeuta faz da autorrevelação, que é uma forma de esse profissional apresentar-se autenticamente a si mesmo ou na relação terapêutica para promover o movimento relacional e o crescimento pessoal do paciente.

Rieger et al. (2010) pontuam que vários estudos oferecem apoio empírico à questão da eficácia da psicoterapia interpessoal no tratamento dos TA. Os autores testaram um modelo de intervenção interpessoal no tratamento dos TA. Esse modelo propõe que a avaliação social negativa desempenha papel central, seja como causa - mediante seu impacto negativo sobre a autoavaliação do paciente -, seja como consequência dos sintomas. Comprovou-se, com o mencionado estudo, que o modelo foi eficaz no tratamento dos TA. Uma alternativa encontrada no campo das psicoterapias aplicadas aos TA é descrita no estudo de Smith, Bartz e Richards (2007), que afirmam que o uso de psicoterapias orientadas espiritualmente aumentou durante a última década. Os resultados da revisão realizada por esses autores evidenciaram que as abordagens de psicoterapia orientadas espiritualmente podem ser benéficas para pessoas com TA. Já Bruno, Rosani e Berlincioni (2009) reportaram bons resultados com a realização de oficinas de teatro com mulheres diagnosticadas com TA, ao mesmo tempo que elas recebiam psicoterapia de grupo de orientação psicanalítica.

Sonnenberg e Chen (2003) discorreram sobre a relevância do aconselhamento de carreira para grupos de pacientes com TA. No referido estudo, incorporaram conceitos teóricos da orientação profissional e do aconselhamento de carreira no processo de ajuda a pacientes com TA. Os autores discutiram as implicações de mesclar o aconselhamento de carreira às intervenções terapêuticas no processo de tratamento. 
Taylor, Cameron, Newman e Junge (2002) descreveram a psicoterapia de base populacional, que considera a prestação de serviços a uma população em risco ou já afetada por uma doença ou transtorno mental. Utilizando dados existentes sobre a prevalência, incidência, fatores de risco e intervenções preventivas e clínicas para TA, o referido estudo examinou questões relacionadas à integração de informações, proporcionando redução de risco e oportunidade de tratamento para uma população de estudantes universitários do sexo feminino, considerando sua vulnerabilidade. Modelos de psicoterapia de base populacional têm implicações importantes para o planejamento da prestação de serviços e para orientar futuras direções em pesquisa sobre alimentação e outros tipos de transtornos e problemas de saúde mental, porém os seus pressupostos devem ser cuidadosamente examinados. Por outro lado, estudos que forneçam dados de base populacional, que combinam redução de fatores de risco e oferta de tratamentos clínicos, são necessários para fazer avançar o conhecimento nesse campo.

Bradshaw, Cooke McDonald (2011) apresentam a metodologia Observed \& Experiential Integration (OEI), desenvolvida como uma inovação clínica em pacientes diagnosticados com diferentes transtornos, entre eles os alimentares. As bases desse tratamento visam a integrar diferentes tradições, como a psicanalítica, comportamental, existencial e a de matriz neurobiológica. Embora seja considerado um tratamento sólido e que vem alcançando bons resultados, ainda é recente sua aplicação no contexto dos TA, como a BN e a AN.

Ainda dentre as abordagens consideradas complementares, destaca-se o estudo de Starzomska (2008), que empregou o correio eletrônico (e-mail) no tratamento dos TA. Alguns estudos recuperados pelo autor sublinham os potenciais benefícios dessa estratégia de intervenção e, para comprovar indiretamente seus efeitos, mencionam opiniões entusiasmadas dos pacientes submetidos a esse modelo de intervenção, o que pode ser entendido como uma limitação metodológica no que concerne à determinação da efetividade do procedimento. Por outro lado, há evidências de que o e-mail pode ajudar alguns pacientes com TA, especialmente os tímidos. Paradoxalmente, o anonimato, que é considerado uma das vantagens do e-mail, pode se tornar o maior perigo para a privacidade dos pa- cientes, caso o sigilo seja violado. Além disso, esse recurso pode ser problemático, no caso de pacientes gravemente perturbados para difundir comportamentos autodestrutivos.

As abordagens complementares revelam que os TA não oferecem um modelo único de tratamento, em razão da pluralidade de modos de compreensão etiopatológica desses quadros e das condições oferecidas pelo sistema de saúde de cada país. As perspectivas teórico-práticas diversificadas influenciam na adoção de modelos e no entendimento de como os sintomas podem ser tratados. Longe de cristalizar uma abordagem "mais adequada" ou "mais aconselhável", destaca-se a existência dessa diversidade como constitutiva do campo dos TA, o que abre inclusive a possibilidade de que outras formas de tratamento possam emergir, quiçá com resultados ainda mais promissores.

\section{Psicoterapias combinadas a outros tratamentos $(\mathrm{n}=9)$}

Nessa categoria são trazidos os estudos que destacam a combinação de técnicas de tratamento dos TA. Há que se destacar que algumas dessas investigações já foram mencionadas anteriormente, embora sejam priorizadas na presente seção por focalizarem a associação de tratamentos para a promoção do bem-estar de pessoas com TA.

Em artigo de atualização do final da década de 1990, Bacaltchuk e Hay (1999) descreveram que as duas abordagens terapêuticas mais estudadas para o tratamento da BN eram, à época, os tratamentos psicológicos, principalmente a TCC, e o uso de antidepressivos. Os resultados de duas revisões sistemáticas da literatura mostraram que a TCC é melhor que fila de espera, e que os antidepressivos são superiores ao placebo na remissão a curto prazo dos sintomas bulímicos. A comparação direta das duas abordagens mostra que, quando utilizadas como recursos exclusivos, a TCC é clinicamente mais eficaz e mais aceita do que os antidepressivos. A associação dos dois tipos de tratamento é clinicamente mais eficaz do que cada um isoladamente, mas, por parte dos pacientes, a aceitação das abordagens psicológicas exclusivas é maior. Porém, como a BN é um transtorno grave e com baixa taxa de remissão espon- 
tânea, o tratamento associado, com abordagem multidimensional e multidisciplinar, é altamente indicado.

Mitchell, Peterson, Myers e Wonderlich (2001) abordam a ideia de combinar a administração de medicamento e psicoterapia no tratamento de pacientes com TA. Na maioria dos casos, a psicoterapia é uma parte essencial do cuidado do paciente, mas a psicofarmacoterapia tem tido um papel crescente nesse cenário. Nos típicos contextos clínicos nos quais os pacientes com TA são vistos, é comum encontrar pessoas com AN ou BN que estão recebendo terapêutica combinada.

Thompson-Brenner, Boisseau e Satir (2010) investigaram, em condições naturalísticas, os resultados do tratamento de 120 adolescentes com TA na comunidade. Após oito meses de tratamento, cerca de um terço dos pacientes havia se recuperado. Pacientes com AN mostraram maiores índices de melhora. Os médicos utilizaram uma série de intervenções psicoterápicas, sendo que dois terços dos pacientes tinham recebido medicação psicoativa.

Bowers e Andersen (2007) referem que a terapia cognitivo-comportamental tem demonstrado eficácia no tratamento da BN, mas há menos dados empíricos sobre sua utilidade com AN ou TCAP. O uso da TCC é recomendado como primeira linha de tratamento para BN, e fortemente recomendado em combinação, quando os medicamentos por si só não se mostram eficazes. Tratamento combinado também melhora sintomas como ansiedade, depressão e restrição alimentar. Dados semelhantes foram referidos na investigação de Pomerantz (2005). Estudo de Hendricks e Thompson (2005) fornece uma ilustração do modelo de integração de TCC e psicoterapia interpessoal para o tratamento da BN, com base em um estudo de caso que compromovou maior eficácia a partir do emprego da TCC.

Comparando os tratamentos ambulatoriais tradicionais e aqueles realizados em regime de hospital-dia, Kong (2005) descobriu que os participantes do programa de tratamento-dia mostraram melhora significativamente maior na maioria dos sintomas psicológicos dos TA. Os dados finais sugerem que os enfermeiros engajados em programas de tratamento-dia podem desempenhar vários papéis importantes e auxiliar a estabelecer uma aliança terapêutica entre paciente e cuidador no período inicial de tratamento. Além disso, o trabalho de inspiração cognitivo-comportamental, que é considera- do vital para a recuperação de quem necessita lidar com dificuldades alimentares, problemas de peso e autoestima, é mais eficaz quando fornecido por uma terapeuta enfermeira que mantenha uma relação empática com o paciente. Zeeck, Herzog e Hartmann (2004) destacam que tratar casos graves de TA em um programa de tratamento-dia tem suas vantagens, mas também apresenta limitações em comparação com o regime de internação integral.

Escobar-Koch et al. (2010) realizaram um estudo transcultural (Estados Unidos e Reino Unido) sobre as perspectivas de usuários dos serviços que atendem pessoas com TA. Ambas as amostras identificaram como aspectos essenciais do cuidado: estabelecimento de boa relação terapêutica, abordagem holística, psicoterapia individual ou aconselhamento, tratamento especializado centrado no paciente, oferta ativa de cuidados e apoio. Na amostra dos Estados Unidos, as principais preocupações referidas foram a falta de acessibilidade aos serviços devido a barreiras financeiras e problemas com a cobertura do seguro-saúde, enquanto que na amostra do Reino Unido foi destacada a falta de igualdade e de disponibilidade de serviços.

Agrupando as contribuições oferecidas nos diversos estudos que compõem o corpus analisado no presente estudo, há que se compreender que, no contexto dos TA, não basta mencionar uma técnica ou modalidade de atendimento considerada como bem-sucedida. Há que se retomar, nesse sentido, quais seriam os critérios preconizados para um tratamento ser considerado adequado ou fortuito. Além disso, algumas abordagens elencam aspectos específicos acerca do que poderia ser considerado como melhora no percurso de enfrentamento do transtorno. Mais do que atestar a assunção dessas diferentes formas e doutrinas de atendimento psicoterápico, há que se promoverem reflexões em torno de aspectos práticos do manejo dos atendimentos que se mostrem mais responsivos às demandas grupais e individuais, explorando o potencial das psicoterapias como espaço privilegiado de interações e autoconhecimento que podem promover a transformação psíquica.

A partir do exposto, um dos focos de investigações futuras poderia ser o manejo do grupo pelos profissionais de saúde, o nível de conhecimento e domínio prático requerido desses profissionais acerca das abordagens empregadas, bem como a possibilidade 
de desenvolver propostas contextualizadas de atendimento que não enquadrem os sintomas dos TA no molde teórico de uma psicopatologia fixa e com tratamento fechado, mas que deem margem a que emerjam outros sentidos sobre a doença e a psicoterapia.

Por fim, há que se considerar que entre os estudos analisados há variabilidade quanto ao controle da pesquisa, número de participantes, delineamentos propostos, índices de confiança, de replicabilidade e de generalização, dentro dos princípios éticos e padrões de evidência vigentes. Por agrupar estudos empíricos, teóricos e de revisão, recomenda-se cautela no uso e na generalização da análise desenvolvida no presente estudo.

\section{Considerações Finais}

A partir do percurso empreendido pela literatura científica, algumas considerações podem ser tecidas. Embora o foco não tenha sido comparar a produção nacional e a internacional, constatou-se que a primeira é menor do que a segunda, o que sugere a necessidade de maior estímulo para que os grupos de pesquisa brasileiros que atuam na área dos TA invistam mais na investigação da psicoterapia e consolidem suas publicações em revistas indexadas.

A pluralidade de técnicas psicoterápicas existentes, que é um dado intrínseco ao campo das psicoterapias, também se apresenta no quadro de tratamento dos TA. Longe de cristalizar um único tipo de tratamento como sendo o mais adequado, o que favoreceria a consideração duvidosa de que haveria uma verdade única e universal em termos de abordagem psicoterapêutica dos TA, alguns relatos de pesquisa conjugam diversas técnicas para restituir o bem-estar do paciente, com a combinação de psicoterapia e medicamentos, por exemplo.

Seja qual for a modalidade psicoterápica priorizada no contexto peculiar de cada serviço especializado, ou a forma mais efetiva de conduzir à adoção de um critério consistente de cura ou melhora do paciente, não se pode perder de vista que essas técnicas são inscritas em determinadas práticas sociais que carregam conceitos, modelos, crenças, valores e aspectos culturais, econômicos e políticos. A maioria dos estudos revisados não traz à baila uma consideração crítica desses determinantes, que contribuiriam para contextualizar os resultados encontrados e evidenciar a necessidade de que os tratamentos se ajustem a demandas específicas, como pouca disponibilidade de recursos, perfil dos pacientes, características dos terapeutas, dos familiares ou mesmo de compreensões sobre corpo, imagem corporal, saúde e bem-estar emocional difundidas e compartilhadas em diferentes tradições. Essas concepções, por sua vez, também não são estanques, mas mutáveis de acordo com o período histórico e o tipo de sociedade. Conclui-se, portanto, que os estudos não se dedicam a explanar os motivos para a adoção de diferentes abordagens ou modalidades de atendimento, de modo que ainda são raras as investigações que apresentam uma reflexão crítica sobre a intervenção analisada.

Um ponto em comum identificado nos artigos revisados, inclusive nos estudos que utilizam uma base psicofarmacológica, é a crença na possibilidade de transformação operada ou coadjuvada pela psicoterapia. A partir disso, pode-se pensar nas potencialidades de favorecer mudanças em um contexto como o dos TA. Embora os profissionais de saúde não tenham sido priorizados nos estudos recuperados, destaca-se que eles - notadamente os psicoterapeutas oriundos da área da Psicologia -, deveriam ser encorajados a superar seus preconceitos e a ultrapassar as fronteiras que muitas vezes separam uma abordagem psicoterápica da outra, e as psicoterapias das abordagens complementares, favorecendo a adoção de posturas menos patologizantes, menos homogêneas e menos estigmatizantes. Assim, poderiam ser incentivadas abordagens mais criativas e abertas à multidiversidade teórico-epistemológica que caracteriza o campo das psicoterapias, tornando-as mais passíveis de transformação.

Dentre as limitações do estudo, destaca-se a seleção exclusiva de artigos, de modo que outras importantes contribuições, advindas principalmente de relatos de experiência profissional, foram excluídas devido às exigências metodológicas da revisão integrativa. Também as contribuições não arbitradas pelo procedimento de revisão de pares poderiam ser recuperadas por outros estudos de revisão. Do modo como foi organizado e apresentado o presente estudo, não houve preocupação com noções de "prevalência" de moda- 
lidades de atendimento ou de abordagens, haja vista que o foco foi colocado na análise crítica da produção científica na área. Em estudos vindouros, abre-se a possibilidade de incluir investigações que se dediquem tanto a mapear a produção na área (o que deve contemplar as mais variadas formas de divulgação científica, presentes em livros, capítulos, dissertações, teses, monografias, anais de congressos, entre outras), como a oferecer sólidas bases para a reflexão de pesquisadores e profissionais que atuam no contexto das psicoterapias e dos TA. Nesse sentido, o foco nos psicoterapeutas pode deflagrar investigações importantes de serem conduzidas. Também se recomenda que outras análises sejam empreendidas, cruzando delineamentos, controles metodológicos e resultados encontrados, o que pode permitir conclusões mais balizadas e que contribuam para a compreensão do estado da arte na área de psicoterapias e TA.

\section{Referências}

Andrade, T. F., \& Santos, M. A. (2009). A experiência corporal de um adolescente com transtorno alimentar. Revista Latino-Americana de Psicopatologia Fundamental, 12 (3), 454-468.

American Psychiatric Association. (2000). Diagnostic and statistical manual of mental disorders ( $4^{\text {th }}$ ed.). Washington, DC: Author.

American Psychiatric Association. (2011). Practice guideline for the treatment of patients with eating disorders. Washington, DC: Author. Retrieved January 2, 2011, from <http://www.psychiatryonline.com/pracGuide/prac GuideChapToc_12.aspx>.

Araújo, M. X., \& Henriques, M. I. R. S. (2011). Que "diferença faz a diferença" na recuperação da anorexia nervosa? Revista de Psiquiatria Clínica, 38 (2), 71-76.

Bacaltchuk, J., \& Hay, P. (1999). Tratamento da bulimia nervosa: síntese das evidências. Revista Brasileira de Psiquiatria, $21(3), 1-6$.

Barth, D. (1991). When the patient abuses food. In H. Jackson (Ed.), Using self psychology in psychotherapy (pp. 223-242). Northvale, NJ: Jason Aronson.

Barth, F. D. (2008). Hidden eating disorders: attachment and affect regulation in the therapeutic relationship. Clinical Social Work Journal, 36 (4), 355-365.

Biasoli-Alves, Z. M. M. (1998). A pesquisa psicológica: análise de métodos e estratégias na construção de um conhecimento que se pretende científico. In G. Romanelli \& Z. M. M. Biasoli-Alves (Orgs.), Diálogos metodológicos sobre prática de pesquisa (pp.135-157). Ribeirão Preto: Legis Summa.
Bowers, W. A., \& Andersen, A. E. (2007). Cognitive-behavior therapy with eating disorders: the role of medications in treatment. Journal of Cognitive Psychotherapy, 21 (1), 16-27.

Bradshaw, R. A., Cook, A., \& McDonald, M. J. (2011). Observed \& Experiential Integration (OEI): discovery and development of a new set of trauma therapy techniques. Journal of Psychotherapy Interaction, 21 (2), 104-171.

Bruno, D., Rosani, M., \& Berlincioni, V. (2009). Giocare al teatro: nota clinica su un'esperienza di laboratorio teatrale con pazienti affette da DCA. Rivista Sperimentale di Freniatria: La Rivista della Salute Mentale, 133 (1), 143-149.

Constantino, M. J., Arnow, B. A., Blasey, C., \& Stewart Agras, W. (2005). The association between patient characteristics and the therapeutic alliance in cognitive-behavioral and interpersonal therapy for bulimia nervosa. Journal of Consulting and Clinical Psychology, 73 (2), 203-211.

Creswell, J. W. (2010). Revisão da literatura. In J. W. Creswell. Projeto de pesquisa (3a ed., pp.48-75). Porto Alegre: Artmed.

Daini, S. (2009). Projective methods in the study of eating disorders: an update. Journal of Projective Psychology \& Mental Health, 16 (2), 117-123.

Escobar-Koch, T., Bancker, J. D., Crow, S., Cullis, J., Ringwood, S., Snieth, G., et al. (2010). Service users' views of eating disorder services: an international comparison. International Journal of Eating Disorders, 43 (6), 549-559.

Fassino, S., Amianto, F., \& Ferrero, A. (2008). Psicoterapia breve psicodinamica adleriana: elementi teorici e indicatori di processo. Minerva Psichiatrica, 49 (3), 203-216.

Fassino, S., Daga, G. A., Pierò, A., \& Rovera, G. G. (2002). Dropout from brief psychotherapy in anorexia nervosa. Psychotherapy and Psychosomatics, 71 (4), 200-206.

Fernandes, M. H. (2006). Transtornos alimentares: anorexia e bulimia. São Paulo: Casa do Psicólogo.

Gaudriault, P., \& Guilbaud, C. (2005). Évolution des boulimiques en psychothérapie dans le test de Rorschach. L'Évolution Psychiatrique, 70 (3), 577-593.

Gowers, S., \& Bryant-Waugh, R. (2004). Management of child and adolescent eating disorders: the current evidence base and future directions. Journal of Child Psychology and Psychiatry and Allied Disciplines, 45 (1), 63-83.

Grave, R. D., Ricca, V., \& Todesco, T. (2001). The stepped-care approach in anorexia nervosa and bulimia nervosa: progress and problems. Eating and Weight Disorders, 6 (2), 81-89.

Grothaus, K. (1998). Eating disorders and adolescents. Journal of Child and Adolescent Psychiatry, 11 (4), 141-51.

Hanns, L. A. (2004). Regulamentação em debate. Ciência e Profissão: Diálogos, 1 (1), 6-13.

Hendricks, P. S., \& Thompson, J. K. (2005). An integration of cognitive-behavioral therapy and interpersonal psychotherapy for bulimia nervosa: a case study using the case formulation method. International Journal of Eating Disorders, 37 (2), 171-174.

Kong, S. (2005). Day treatment programme for patients with eating disorders: randomized controlled trial. Journal of Advanced Nursing, 51 (1), 5-14. 
Leichsenring, F. (2005). Are psychodynamic and psychoanalytic therapies effective? A review of empirical data. International Journal of Psychoanalysis, 86 (3), 841-868.

Lewis, A. J., Dennerstein, M., \& Gibbs, P. M. (2008). Shortterm psychodynamic psychotherapy: review of recent process and outcome studies. Australian and New Zealand Journal of Psychiatry, 42 (6), 445-455.

Lock, L. (2010). Treatment of adolescent eating disorders: progress and challenges. Minerva Psichiatrica, 51 (3), 207-216.

Messer, S. B. (2001). What makes brief psychodynamic therapy time efficient? Clinical Psychology, 8 (1), 5-22.

Mitchell, J. E., Peterson, C. B., Myers, T., \& Wonderlich, S. (2001). Combining pharmacotherapy and psychotherapy in the treatment of patients with eating disorders. Psychiatric Clinics of North America, 24 (2), 315-323.

Moro, M. (2001). Un'esperienza di formazione in psicoterapia di gruppo per la terapia delle patologie del comportamento alimentare. Psicoterapia e Istituzioni: Review of Psychoanalytical Methodological Research and Clinical Experiences, 8 (2), 79-81.

Organização Mundial da Saúde (2002). Relatório Mundial da Saúde: saúde mental: nova concepção, nova esperança. Lisboa: Ministério da Saúde. Recuperado em agosto 15, 2011, disponível em <http://www.who.int./whr>

Peres, R. S., \& Santos, M. A. (2011). Técnicas projetivas na avaliação de aspectos psicopatológicos da anorexia e bulimia. Psico - USF, 16 (2), 185-192.

Pinto, F. P., Santeiro, T. V., \& Santeiro, F. R. M. (2010). Produção científica sobre psicoterapias na base de dados PePSIC (1998/2007). Fractal: Revista de Psicologia, 22 (2), 411-430.

Pomerantz, J. M. (2005). Eating disorders: when is medication use appropriate? Drug Benefit Trends, 17 (8), 364-365.

Ramacciotti, A., Sorbello, M., Pazzagli, A., Vismara, L., \& Mancone, P. S. (2001). Attachment processes in eating disorders. Eating and Weight Disorders, 6 (3), 166-170.

Rieger, E., Van Buren, D. J., Bishop, M., Tanofsky-Kraff, M., Welch, R., \& Wilfley, D. E. (2010). An eating disorderspecific model of interpersonal psychotherapy (IPT-ED): causal pathways and treatment implications. Clinical Psychology Review, 30 (4), 400-410.

Rosa, B. P., \& Santos, M. A. (2011). Comorbidade entre bulimia e transtorno de personalidade borderline: implicações para o tratamento. Revista Latinoamericana de Psicopatologia Fundamental, 14 (2), 268-282.

Ruiz-Lázaro, P. M. (2003). Terapia familiar hospitalaria ante un trastorno alimentario. Revista de Psiquiatría Infanto-Juvenil, 20 (1), 20-28.

Rutherford, L., \& Couturier, J. (2007). A review of psychotherapeutic interventions for children and adolescents with eating disorders. Journal of the Canadian Academy of Child and Adolescent Psychiatry, 16 (4), 153-157.

Salierno, F. (2001). Un'esperienza da "osservatrice" in un gruppo di genitori di pazienti con disturbi del comportamento alimentare. Psicoterapia e Istituzioni:
Review of Psychoanalytical Methodological Research and Clinical Experiences, 8 (2), 93-96.

Santeiro, T. V. (2008). Psicoterapia breve psicodinâmica preventiva: pesquisa exploratória de resultados e acompanhamento. Psicologia em Estudo, 13 (4), 761-770.

Santos, M. A. (2007). Psicoterapia psicanalítica: aplicações no tratamento de pacientes com problemas relacionados ao uso de álcool e outras drogas. SMAD: Revista Eletrônica Saúde Mental, Álcool e Drogas, 3 (1), 1-15.

Santos, M., \& Zaslavsky, J. (2007). Pesquisando conceitos e tendências em psicoterapia e psicanálise. Revista Brasileira de Psicanálise, 41 (2), 115-124.

Scorsolini-Comin, F. (2010). Metodologia de pesquisa: uma abordagem científica e aplicada. Ribeirão Preto: Instituto de Ensino e Pesquisa em Administração.

Scorsolini-Comin, F., Souza, L. V., \& Santos, M. A. (2010). A construção de si em um grupo de apoio para pessoas com transtornos alimentares. Estudos em Psicologia (Campinas), 27 (4), 467-478. doi: 10.1590/S0103-166X2 010000400005

Sicchieri, J. M. F., Santos, M. A., Dos Santos, J. E., \& Ribeiro, R. P. P. (2007). Avaliação nutricional de portadores de transtornos alimentares: resultados após a alta hospitalar. Ciência, Cuidado e Saúde, 6 (1), 68-75.

Smith, T. B., Bartz, J., \& Richards, P. S. (2007). Outcomes of religious and spiritual adaptations to psychotherapy: a meta-analytic review. Psychotherapy Research, 17 (6), 643-655.

Sonnenberg, S. L., \& Chen, C. P. (2003). Using career development theories in the treatment of clients with eating disorders. Counselling Psychology Quarterly, 16 (2), 173-185.

Souza, L. V. (2011). Construindo cuidado: a relação com os profissionais da saúde nas práticas discursivas de pessoas diagnosticadas com transtornos alimentares. Tese de doutorado não-publicada, Programa de Pós-Graduação em Psicologia, Universidade São Paulo, Ribeirão Preto.

Souza, L. V., \& Santos, M. A. (2010). A participação da família no tratamento dos transtornos alimentares. Psicologia em Estudo, 15 (2), 285-294.

Starzomska, M. (2008). Perspective of the use of electronic communication in the psychotherapy of eating disorders. Archives of Psychiatry and Psychotherapy, 10 (1), 55-65.

Sydow, K., Beher, S., Schweitzer, J., \& Retzlaff, R. (2010). The efficacy of systemic therapy with adult patients: a metacontent analysis of 38 randomized controlled trials. Family Process, 49 (4), 457-485.

Tantillo, M. (2004). The terapist's use of self-disclosure in a relational therapy approach for eating disorders. Eating Disorders: The Journal of Treatment \& Prevention, 12 (1), $51-73$.

Tasca, G. A., Ritchie, K., \& Balfour, L. (2011). Implications of attachment theory and research for the assessment and treatment of eating disorders. Psychotherapy: Theory, Research, Practice, Training, 1 (1), 1-11.

Taylor, C. B., Cameron, R. P., Newman, M. G., \& Junge, J. (2002). Issues related to combining risk factor reduction 
and clinical treatment for eating disorders in defined populations. The Journal of Behavioral Health Services \& Research, 29 (1), 81-90.

Teriato, V. (2009). Dal cibo alle fantasie: II trattamento psicoterapeutico dei disturbi del comportamento alimentare nell'adolescenza. Infanzia e Adolescenza, 8 (1), 35-44.

Thompson-Brenner, H., Boisseau, C. L., \& Satir, D. (2010). Adolescent eating disorders: treatment and response in a naturalistic study. Journal of Clinical Psychology, 66 (3), 277-301.

Toffoli, G. A., \& Ferreira, S. M. S. P. (2011). Mapeamento da produção científica de pesquisadores brasileiros de ciências da comunicação: período de 2000 a 2009. Psicologia USP, 22 (2), 399-422.

Weinberg, C., \& Berlinck, M. T. (2010). A hiperatividade das anoréxicas: uma defesa maníaca? In A. P. Gonzaga \& C.
Weinberg (Orgs.), Psicanálise de transtornos alimentares (pp.102-119). São Paulo: Primavera Editorial.

Weissman, M. M., Markowitz, J. C., \& Klerman, G. L. (2009). Psicoterapia interpessoal: guia prático do terapeuta. Porto Alegre: Artmed.

Zeeck, A., Herzog, T., \& Hartmann, A. (2004). Day clinic or inpatient care for severe bulimia nervosa? European Eating Disorders Review, 12 (2), 79-86.

Zerbe, K. J. (2001). The crucial role of psychodynamic understanding in the treatment of eating disorders. Psychiatric Clinics of North America, 24 (2), 305-313.

Recebido em: 16/11/2011

Versão final em: 30/5/2012

Aprovado em: 20/6/2012

863 
